\title{
Cambio de comportamiento en personas fumadoras posimplementación de legislación antitabaco en Argentina
}

\author{
Guillermo Gabriel Barrenechea, ${ }^{1}$ Cosme Marcelo Furtado Passos da Silva ${ }^{2}$ y \\ Valeska Carvalho Figueiredo ${ }^{3}$
}

Forma de citar

Barrenechea GG, Furtado Passos da Silva CM, Carvalho Figueiredo V. Cambio de comportamiento en personas fumadoras posimplementación de legislación antitabaco en Argentina. Rev Panam Salud Publica. 2019;43:e5. https://doi.org/10.26633/RPSP.2019.5

RESUMEN Objetivo. Identificar factores asociados al cambio de comportamiento en personas fumadoras mayores de 18 años luego de la implementación de políticas provinciales 100\% libre de humo en Argentina.

Métodos. Se realizó un estudio analítico de corte transversal. Se utilizó la técnica de modelos multiniveles trabajando con datos de distintas jerarquías. Se utilizaron fuentes de datos secundarios: Encuesta Nacional de Factores de Riesgo 2005, 2009 y 2013.

Resultados. En el análisis individual de las variables explicativas, se obtuvo que los pobladores de las provincias que no tienen legislación tuvieron un $40 \%$ más de probabilidad de no pensar en dejar de fumar que las personas que viven en provincias con legislación. En las provincias sin legislación, el sexo masculino y la edad mayor de 65 años son las características que aumentan las chances de las personas a no pensar en dejar de fumar, en comparación con las provincias que tiene legislación.

Conclusiones. Los resultados sugieren que una legislación basada en el artículo 8 del Convenio Marco resultaría efectiva para estimular la cesación tabáquica en Argentina.

Palabras clave Tabaco; comportamiento; análisis multinivel; política de salud; salud pública; Argentina.

Dirección de Epidemiología, Ministerio de Salud Pública, Tucumán, Argentina. Enviar correspondencia a Guillermo Barrenechea, barrenecheagg@ gmail.com

2 Departamento de Epidemiologia e Métodos Quantitativos em Saúde, Escola Nacional de Saúde Pública Sergio Arouca. Fundação Oswaldo Cruz, Rio de Janeiro, Brasil.

3 Centro de Estudos sobre Tabaco e Saúde e Departamento de Epidemiologia, Escola Nacional de Saúde Pública Sergio Arouca, Fundação Oswaldo Cruz, Rio de Janeiro, Brasil.

Los efectos nocivos del tabaquismo sobre la salud son conocidos y constituyen la base para controlar su consumo. Tanto a nivel global como en el ámbito local existe evidencia científica que permite entender al tabaco como productor de enfermedad y causa de muerte $(1,2)$.

En respuesta a la epidemia mundial de tabaquismo, se impulsó el Convenio Marco para el Control de Tabaco de la Organización Mundial de la Salud (CMCT-OMS), cuyo objetivo es proteger a la población de los efectos negativos del tabaquismo, la exposición al humo de tabaco y la prevalencia de consumo. El CMCT-OMS está firmado por 180 países, lo que lo convierte en el tratado más respaldado de toda la historia (3).
El artículo 8 del CMCT-OMS propone que los países deben asumir la responsabilidad de proteger a la población contra la exposición al humo de tabaco. La legislación establece la implementación de ambientes $100 \%$ libres de humo en los espacios cerrados de acceso público, lugares de trabajo y transporte público, sin excepciones. Es la única medida que garantiza que todas las personas estén protegidas de los efectos de la exposición al humo de tabaco. Aquellos países que prohibieron fumar en ambientes públicos cerrados presentaron reducción de la

Este es un artículo de acceso abierto distribuido bajo los términos de la licencia Creative Commons Attribution-NonCommercial-NoDerivs 3.0 IGO, que permite su uso, distribución y reproducción en cualquier medio, siempre que el trabajo original se cite de la manera adecuada. No se permiten modificaciones a los artículos ni su uso comercial. Al reproducir un artículo no debe haber ningún indicio de que la OPS o el artículo avalan a una organización o un producto específico. El uso del logo de la OPS no está permitido. Esta leyenda debe conservarse, junto con la URL original del artículo. 
mortalidad por enfermedades cardiovasculares y de la prevalencia de tabaquismo (4). Un ejemplo de ello es la revisión sistemática y metaanálisis que realizó un análisis cuantitativo de la reducción del riesgo de infarto agudo de miocardio luego de la implementación de este tipo de legislaciones, además de la disminución de la prevalencia del tabaquismo. Los resultados demostraron que legislaciones de ambientes 100\% libres de humo se asociaron con una reducción significativa en el riesgo de infarto agudo de miocardio (5).

Para ayudar a los países a cumplir el Convenio Marco, la OMS ha establecido la estrategia MPOWER, un plan integrado por las seis medidas más importantes y eficaces de control del tabaco (6).

\section{Legislación en América y Argentina}

Desde 2005, los países de América Latina utilizan dos estrategias efectivas diferentes para convertirse en $100 \%$ libre de humo: la legislación nacional y la legislación subnacional. Una ley de ambiente $100 \%$ libre de humo de significa que el consumo de tabaco está prohibido en todos los lugares públicos cerrados, lugares de trabajo (incluidos restaurantes y bares) y transporte público, sin excepción alguna. Sin embargo, las tabacaleras se encargan de que este tipo de leyes no se aprueben y que sí se aprueben aquellas que dejan excepciones en su enunciado, como la generación de espacios para fumadores, la elección voluntaria de los dueños de los lugares para elegir si se convierten en libres de humo o no, etc. En la actualidad, existe evidencia clara de que la mayoría de las personas están a favor de la prohibición, ya sean fumadores, exfumadores o que nunca hayan fumado (7).

En 2006, Uruguay se convirtió en el primer país de América Latina en adoptar como política nacional la de ambientes $100 \%$ libres de humo. En 2012, otros seis países lo siguieron (Colombia y Panamá en 2008, Perú en 2010, Brasil y Venezuela en 2011 y Costa Rica en 2012). Por otro lado, Argentina, al igual que en Estados Unidos, Canadá y Australia y otros países de América Latina implementan leyes de ambientes libres de humo a nivel subnacional (por ejemplo, a nivel provincial o municipal); por este motivo, algunas provincias cuentan con políticas de ambientes $100 \%$ libres de humo y otras, no. A su vez, las tabacaleras también se encargaron de debilitar o bloquear las leyes que surgen en las distintas provincias.

Argentina ya tenía una ley nacional sancionada en 1986, la cual reglamentaba la publicidad del tabaco, cigarros y cigarrillos (8). En el año 2011, se establece una ley más amplia y acorde al CMCT, en la que se menciona la prohibición de fumar en lugares cerrados, pero en uno de sus artículos plantea algunas excepciones (9). Esta ley nacional, aprobada en 2011, fue reglamentada recién en 2013. Las provincias elaboran y reglamentan leyes provinciales a partir del año 2005. El carácter de cada ley provincial está condicionado por su contenido y puede tener excepciones o no. En las provincias en las que existen excepciones, se considera que la ley es débil. En aquellos casos en los que la ley no hace excepción alguna sobre los ambientes libres de humo, se la considera fuerte. En Argentina, esta estrategia favoreció la disminución de algunos indicadores, como el descenso de la prevalencia de tabaquismo y la disminución en la exposición de humo de tabaco ajeno (10).

\section{Efecto de la legislación sobre el comportamiento}

Para evidenciar el impacto de este tipo de leyes, resulta interesante trabajar con variables relacionadas con el cambio de actitud de las personas, condicionado por factores individuales y de su entorno. Es por ello que las variables relacionadas con el comportamiento juegan un rol importante. En el estudio de cohortes prospectiva, el International Tobacco Control Four Country Survey (ITC-4) evaluó el impacto de la implementación de la ley en cuatro países, tanto desde el punto de vista psicosocial como en el comportamiento de las personas. La evaluación se realizó mediante una serie de factores predictores para dejar de fumar, de los cuales la motivación para dejar de fumar es el predictor más fuerte para lograrlo (11). Por otro lado, no encontró diferencia entre las personas de uno $u$ otro sexo para el abandono. No se encontró asociación entre dejar de fumar y la edad como predictor; sin embargo existen estudios que mencionan que la edad actuaría como predictor (12). También hay estudios que hallaron evidencias sobre el cambio de actitud de las personas luego de la implementación de una ley nacional. En Finlandia, se evaluó la actitud de empleados del sector industrial, de servicios y oficinas del área metropolitana, donde se observó, tanto en fumadores como en no fumadores, un cambio hacia la prohibición total de fumar en esos espacios (13). Otro ejemplo es el estudio realizado en dos universidades de Estados Unidos de América, donde se evaluó el impacto de una política de campus libres de humo. Luego de la implementación, se observó un cambio significativo en la actitud de los estudiantes hacia la regulación del consumo de tabaco (14). La implementación de este tipo de políticas puede generar un cambio de actitud en las personas y llevarlas a tener ciertas reglas dentro de sus hogares, lo cual se traduciría en un menor consumo y en menor exposición a humo de segunda mano. Esto modificaría el comportamiento de todo el grupo, pero sobre todo de los jóvenes (15-17). También existe evidencia de que, además de ser necesarias, las políticas deben ir acompañadas del compromiso de los profesionales de la salud, quienes juegan un rol importante en la aplicación de intervenciones para un cambio en la actitud de las personas fumadoras (18).

En este trabajo se pretende obtener una medida concreta de la implementación de una ley y cómo puede condicionar un cambio en el comportamiento de las personas. Es por ello que el objetivo de este estudio fue identificar factores asociados al cambio de comportamiento en personas fumadoras mayores de 18 años después de la implementación de la ley $100 \%$ libre de humo en Argentina.

\section{MATERIALES Y MÉTODOS}

Se realizó un estudio de corte transversal, se utilizaron tres puntos en el tiempo. Se utilizó la técnica de modelos multiniveles que trabajan con datos de distintas jerarquías. En este tipo de estudios se puede utilizar información tanto acerca de los individuos como de su contexto. Por lo tanto, la información disponible tiene distintas jerarquías: el nivel 1, individual, y el nivel 2, que corresponde al contexto o grupo al que pertenece el individuo, en este caso la provincia en la que vive (19). Al establecer esta jerarquía entre las distintas variables, se dice que los individuos 
pertenecientes a un mismo contexto tenderán a ser más similares en su comportamiento; por ejemplo, aquellas personas que viven en la misma provincia con la misma legislación podrían tener comportamientos más parecidos entre sí.

Para establecer si hubo cambios en la conducta tabáquica (voluntad de abandonar el consumo de tabaco), se utilizó como fuente secundaria la Encuesta Nacional de Factores de Riesgo 2005, 2009 y 2013 (20-22). Dichas encuestas fueron validadas en el año 2004, fueron domiciliarias y tienen representatividad provincial. El diseño muestral fue probabilístico multietápico. La población objetivo para esta encuesta comprende las personas de 18 años y más, que residen en hogares particulares en localidades de 5000 y más habitantes de la República Argentina.

Para el análisis de los datos, se unieron las tres bases de datos correspondientes a las tres encuestas (año 2005, $\mathrm{n}=41$ 392; año 2009, $\mathrm{n}=34$ 732; año $2013, n=32$ 365). Se nominalizó y se recategorizaron las variables para que los nombres y categorías coincidieran en las tres encuestas. Se calcularon proporciones con sus respectivos intervalos de confianza del 95\% (IC95\%) para obtener la distribución de la variable respuesta en los distintos grupos estudiados. La variable respuesta utilizada para cumplir el objetivo (piensa en dejar de fumar: Sí o No) tiene una distribución binomial. Para el análisis descriptivo y el modelo logístico multinivel, se utilizó la función "glmer" de la biblioteca "lme4" del software $\mathrm{R}^{\circledR}$ (23). En los test se utilizó un nivel de significancia de 5\%. Se seleccionaron las variables que resultaron significativas en los modelos bivariados y fueron incluidas en el modelo completo. Todas las variables del modelo completo presentaron al menos una categoría significativa.

Las variables independientes que contempla la encuesta son:

- Sexo: mujer, hombre.

- Rangos de edad en años: 18-24, 25-34, 35-49, 50-64, 65 y más.

- Condición de actividad: ocupado (persona que tiene por lo menos una ocupación, es decir que en la semana de referencia ha trabajado como mínimo una hora en una actividad económica) desocupado (persona que, sin ocupación, está en búsqueda activa de trabajo), inactivo (persona que no tiene trabajo ni lo busca en forma activa, se subdividen en inactivos marginales e inactivos típicos, según estén dispuestos o no a trabajar).

- Tipo de vivienda: es el tipo de unidad habitacional donde reside la persona encuestada. Se agruparon las categorías según si la persona encuestada era dueña o no del espacio donde residía: casa (dueño o propietario de la vivienda donde reside), departamento (no es dueño), otros (inquilinos, hotel, casilla, etc.).

- Nivel de instrucción: grado de instrucción alcanzado por la persona encuestada: primario incompleto, primario completo, secundario incompleto, secundario completo y más).

- Año de realización de la encuesta: 2005, 2009 o 2013.

Como variables de segundo nivel se utilizó la clasificación de las provincias según el contenido de la ley. Se consideró como provincias con presencia de ley aquellas provincias que tienen leyes $100 \%$ libre de humo sin excepciones. Aquellas provincias que tienen una ley con algunas excepciones en su contenido y son consideradas leyes débiles o de carácter medio se tomaron como ausencia de ley. De esta manera, quedó una variable dicotómica: presencia (ley jurisdiccional implementada de carácter fuerte sin excepciones) o ausencia (implementación de leyes de carácter débiles, medio o no implementación de leyes jurisdiccionales).

En cuanto a la variable dependiente, se evaluó la conducta de las personas que fumaban en base a la pregunta si pensaban en dejar de fumar. Se adoptó a aquellas personas que sí lo hacían como la categoría de referencia y a las que no pensaban en dejar de fumar como la categoría de riesgo: pensar en dejar de fumar (sí o no).

Para la selección del modelo que mejor explique si la actitud de las personas en pensar en dejar de fumar depende de las condiciones de su entorno y si la ley resulta significativa para condicionar ese cambio de comportamiento, se elaboraron modelos logísticos multiniveles con cada una de las variables seleccionadas de la encuesta y elaboradas a partir de la legislación vigente en cada una de las provincias.

Entre las consideraciones éticas que se tuvieron en cuenta al realizar el estudio, dado que este es de carácter observacional, no implica intervenciones ni contacto directo con pacientes o personas. Se respetó en todo momento la confidencialidad de los datos según la Ley Nacional 25326 de Protección de Datos Personales de Argentina. En ese sentido, el presente estudio utilizó datos no vinculables, los cuales son información de conocimiento público. Por otro lado, el estudio utilizó registros sanitarios con reconocimiento oficial por parte de las autoridades sanitarias en los cuales no es posible establecer la identidad de las personas. Aun así, el proyecto fue sometido y aprobado por el Comité de Ética en Investigación del Instituto Nacional de Epidemiología.

\section{RESULTADOS}

\section{Análisis descriptivo}

A continuación, se muestra los principales resultados de la estadística descriptiva (cuadro 1). De ellos se desprende que existen diferencias en la frecuencia de personas que no piensan en dejar

CUADRO 1. Distribución de frecuencias de la variable respuesta según variables independientes seleccionadas

\begin{tabular}{|c|c|c|}
\hline \multirow[t]{2}{*}{ Variables explicativas } & \multicolumn{2}{|c|}{$\begin{array}{c}\text { Piensa en dejar } \\
\text { de fumar }(\%)\end{array}$} \\
\hline & Sí & No \\
\hline \multicolumn{3}{|l|}{ Sexo } \\
\hline Mujer & 63 & 37 \\
\hline Hombre & 61 & 39 \\
\hline \multicolumn{3}{|l|}{ Año de la encuesta } \\
\hline 2005 & 52 & 48 \\
\hline 2009 & 81 & 19 \\
\hline 2013 & 61 & 39 \\
\hline \multicolumn{3}{|l|}{ Grupos de edad (años) } \\
\hline $18-24$ & 60 & 40 \\
\hline $25-34$ & 62 & 38 \\
\hline $35-49$ & 60 & 40 \\
\hline $50-65$ & 60 & 40 \\
\hline 65 y más & 51 & 49 \\
\hline \multicolumn{3}{|l|}{ Condición de actividad } \\
\hline Ocupado & 60 & 40 \\
\hline Desocupado & 63 & 37 \\
\hline Inactivo & 58 & 42 \\
\hline \multicolumn{3}{|l|}{ Nivel de instrucción } \\
\hline Primario incompleto & 56 & 44 \\
\hline Secundario incompleto & 60 & 40 \\
\hline Secundario completo y más & 60 & 38 \\
\hline \multicolumn{3}{|l|}{ Tipo de vivienda } \\
\hline Casa & 60 & 40 \\
\hline Departamento & 62 & 38 \\
\hline Otros & 63 & 37 \\
\hline
\end{tabular}


CUADRO 2. Porcentaje de personas que no piensan en dejar de fumar, por provincia y según el año de realización de la encuesta

\begin{tabular}{lcc}
\hline \multirow{2}{*}{ Provincia } & \multicolumn{2}{c}{$\begin{array}{c}\text { Año de la } \\
\text { encuesta }\end{array}$} \\
\cline { 2 - 3 } & 2005 & 2013 \\
\hline C.A.B.A. & 41 & 31 \\
Buenos Aires & 45 & 34 \\
Catamarca & 41 & 42 \\
Córdoba & 50 & 32 \\
Corrientes & 42 & 29 \\
Chaco & 64 & 47 \\
Chubut & 49 & 41 \\
Entre Ríos & 53 & 36 \\
Formosa & 36 & 37 \\
Jujuy & 68 & 44 \\
La Pampa & 44 & 43 \\
La Rioja & 47 & 33 \\
Mendoza & 51 & 43 \\
Misiones & 60 & 21 \\
Neuquén & 47 & 35 \\
Río Negro & 42 & 45 \\
Salta & 51 & 38 \\
San Juan & 41 & 49 \\
San Luis & 47 & 36 \\
Santa Cruz & 45 & 45 \\
Santa Fe & 52 & 40 \\
Santiago del Estero & 47 & 47 \\
Tucumán & 55 & 50 \\
Tierra del Fuego & 37 & 35 \\
\hline CA.BA, Ciuda Ánno & &
\end{tabular}

C.A.B.A., Ciudad Autónoma de Buenos Aires.

de fumar según categorías de variables independientes seleccionadas de la encuesta. Entre los resultados más relevantes, se observó que los más propensos a no pensar en dejar de fumar son aquellos que pertenecen al grupo de edad más extremo, los que poseen una situación habitacional más vulnerable y una mejor situación laboral; se observa una relación inversa con nivel de instrucción.

En cuanto al análisis descriptivo de la variable respuesta según provincias, se observa que, en 2005, las provincias que presentaban mayores porcentajes de individuos que no pensaban en dejar de fumar fueron Jujuy y Misiones, que son provincias productoras de tabaco (cuadro 2).

\section{Análisis multinivel}

La ausencia de ley fue la variable con más fuerte asociación a no pensar en dejar de fumar (cuadro 3). El grupo de inactivos está formado por estudiantes y jubilados en su mayoría. Por otro lado, se

CUADRO 3. Razón de probabilidades no ajustadas para proporción de fumadores que no piensan en dejar de fumar según variables sociodemográficas y el año de encuesta

\begin{tabular}{llll}
\hline Variables & Categorías & RP & IC95\% \\
\hline Ley & Presencia & 1,00 & \\
Sexo & Ausencia & 1,40 & $1,06-1,86$ \\
& Mujeres & 1,00 & \\
Rango de edad (años) & Hombres & 1,08 & $1,02-1,13$ \\
& $18-24$ & 1,00 & \\
& $25-34$ & 0,94 & $0,87-1,02$ \\
& $35-49$ & 1,00 & $0,93-1,08$ \\
Tipo de vivienda & $50-64$ & 1,01 & $0,93-1,10$ \\
& 65 y más & 1,47 & $1,31-1,66$ \\
Condición de actividad & Casa & 1,00 & \\
& Departamento & 0,94 & $0,88-1,02$ \\
Nivel de instrucción & Otros & 0,87 & $0,77-1,00$ \\
& Ocupado & 1,00 & \\
& Desocupado & 0,88 & $0,79-0,98$ \\
Año de la encuesta & Inactivo & 1,09 & $1,02-1,16$ \\
& Primario incompleto & 1,00 & \\
& Primario completo & 0,88 & $0,81-0,96$ \\
& Secundario completo y más & 0,82 & $0,75-0,89$ \\
\hline & 2005 & 1,00 & \\
& 2009 & 0,25 & $0,23-0,27$ \\
& 2013 & 0,68 & $0,64-0,72$ \\
\hline
\end{tabular}

RP, razón de probabilidades; IC95\%, intervalo de confianza de $95 \%$.

Fuente: elaboración propia.

CUADRO 4. Razón de probabilidades ajustadas mediante análisis multinivel para la proporción de fumadores que no piensan en dejar de fumar, según variables sociodemográficas y el año de la encuesta

\begin{tabular}{llcc}
\hline Variables & Categorías & RP & IC95\% \\
\hline Ley & Presencia & 1,00 & \\
Sexo & Ausencia & 1,39 & $1,05-1,84$ \\
& Mujeres & 1,00 & \\
Rango de edad (años) & Hombres & 1,08 & $1,02-1,14$ \\
& $18-24$ & 1,00 & \\
& $25-34$ & 0,97 & $0,90-1,05$ \\
& $35-49$ & 1,00 & $0,92-1,08$ \\
& $50-64$ & 1,03 & $0,94-1,12$ \\
Condición de actividad & Ocupado & 1,48 & $1,30-1,68$ \\
& Desocupado & 1,00 & \\
Nivel de instrucción & Inactivo & 0,88 & $0,78-0,98$ \\
& Primario incompleto & 1,05 & $0,98-1,12$ \\
Año de la encuesta & Secundario incompleto & 1,00 & \\
& Secundario completo y más & 0,93 & $0,85-1,02$ \\
& 2005 & 0,86 & $0,79-0,94$ \\
& 2009 & 1,00 & \\
& 2013 & 0,24 & $0,22-0,27$ \\
\hline
\end{tabular}

RP, razón de probabilidades; IC95\%, intervalo de confianza de $95 \%$.

Fuente: elaboración propia.

observa que el aumento en el nivel de instrucción ejerce mayor efecto protector en el nivel de instrucción.

Para la construcción del modelo completo no se tuvieron en cuenta las variables que no fueron significativas a nivel individual (cuadro 3). Al obtener el modelo logístico multinivel completo, las asociaciones se mantienen en magnitud y en signo, lo cual concuerda y confirma los resultados obtenidos en el análisis individual (cuadro 4). 


\section{DISCUSIÓN}

Los resultados de este estudio resultan una evidencia empírica del impacto del artículo 8 del CMCT en el estímulo para dejar de fumar y la reducción de la prevalencia del tabaquismo al indicar que la implementación de leyes de ambientes libres de humo produce un cambio en la forma de pensar de las personas que puede llevarlas a tratar de dejar de fumar. Cuando se analiza de forma individual, se observa que los varones, las personas de más de 65 años, las que poseen un trabajo y los de menor escolaridad son las características que llevarían a las personas a no pensar en dejar de fumar. Esto indicaría los posibles nichos en los cuales la ley no haría impacto o hacia los cuales se deberían dirigir las estrategias. Se destaca que aquellas personas que viven en provincias que no han implementado una ley fuerte tienen un 39\% más de probabilidades de no pensar en dejar de fumar. Otro punto importante es que, a medida que pasa el tiempo, las probabilidades de no pensar en dejar de fumar son menores a la medida inicial, tomada en el año 2005.

Las políticas de control del tabaco como las leyes de aire libre de humo están diseñadas para proteger a los no fumadores y desalentar el tabaquismo entre los fumadores. En una revisión sistemática donde se evaluó el efecto de la ley de espacios libres de humo sobre el comportamiento de los fumadores, se halló que la implementación de este tipo de políticas se asocia con una disminución de $3,8 \%$ en la prevalencia y $3,1 \%$ en el consumo de cigarrillos (24). Además, también tiene efecto sobre las actitudes de las personas que tienden apoyar a la restricción de fumar dentro de lugares cerrados $y$, de esa manera, disminuye la exposición a jóvenes y niños (25). Es necesario que estas políticas tengan apoyo desde todos los niveles; un ejemplo pueden ser los programas de viviendas desarrollados con fondos públicos, en lo que se construyen las viviendas desde el inicio bajo el concepto de libres de humo (26).

La mayoría de las investigaciones sugieren que las políticas y los programas pueden influir y llevar a las personas a dejar de fumar; sin embargo, son pocas las que se enfocan en estudiar el comportamiento de las personas bajo diferentes estrategias de control de tabaco dentro de un mismo país. En un estudio se exploró la asociación entre el comportamiento de adolescentes con respecto al tabaquismo y las políticas de control de tabaco, donde se utilizaron modelos jerárquicos para vincular los datos de los individuos con los del estado al cual pertenecían (27). La voluntad de dejar de fumar mostró una asociación significativa con el aumento en el precio de los cigarrillos como medida a nivel estatal. Esto demostraría la necesidad de incluir este tipo de medidas en las leyes de control del tabaquismo, punto que no está incluido en la ley nacional.

En un estudio transversal realizado en India se utilizó análisis multinivel y se demostró que el consumo de tabaco tiene una distribución socioeconómica y espacial en ese país; la población en peor situación están en mayor riesgo de consumir tabaco (28). En ese estudio se observó un fuerte gradiente entre educación y tabaquismo, con mayores probabilidades de ser un fumador para el grupo de peor educación (analfabetos) con respecto al grupo de mayor educación (personas con formación de posgrado). Algo similar surgió de los resultados de nuestro estudio, en donde las personas de menor educación presentan menor probabilidad de pensar en dejar de fumar. Esto indicaría que el grupo de menor instrucción tiene menos concientización de los problemas de salud que trae aparejado el tabaquismo.

Existe evidencia del cambio en el comportamiento de las personas a dejar de fumar al implementar este tipo de legislación. En nuestro caso, encontramos evidencia similar que indica que las personas que viven en provincias sin ley tienen menos probabilidad de dejar de fumar. También hay reportes que hablan de la importancia de la profundidad de la infraestructura de la implementación de la legislación y el esfuerzo por parte de los gobiernos locales (29).

Entre las fortalezas del presente estudio se halla el aporte de información sobre la relación entre la aplicación de la estrategia subnacional de políticas de control de tabaco en Argentina y el comportamiento de personas fumadoras.

Entre las limitaciones, se mencionan las correspondientes a los estudios ecológicos. En el caso de este estudio, se intenta contrarrestarlas al trabajar con análisis multinivel, que permite permite obtener resultados teniendo en cuenta variables a nivel individual y provincial, asumiendo que los individuos de la misma provincia están bajo el efecto de la misma ley.
Gran parte de los estudios se encargan de investigar las brechas en el consumo de tabaco, para determinar cuáles son las personas o grupos que son más propensos a consumir tabaco. A diferencia de ese grupo de estudios, en este trabajo se aplicaron los procedimientos estadísticos multinivel para modelar la variación en la intención de abandonar el consumo de tabaco a diferentes niveles. Esto permitió describir cuáles son los grupos más conscientes que piensan en dejar de fumar y ver también cuáles son los grupos más afectados en el sentido de no tener en mente la posibilidad de dejar de fumar.

\section{CONCLUSIÓN}

Los resultados muestran que las personas que pertenecen a una determinada jurisdicción que tiene implementada la ley tiende a cambiar su comportamiento en favor de pensar en dejar de fumar. La comprensión del efecto de las políticas sobre la conducta de las personas podía ayudar a aclarar cuáles son los puntos de mayor influencia y así obtener resultados más efectivos.

Entre las recomendaciones se destaca la necesidad de documentar y vigilar de manera sistemática las variaciones del consumo de tabaco como en el comportamiento de las personas frente al tabaquismo para entender mejor sus determinantes, y para proporcionar una base científica a las personas encargadas de las intervenciones en salud.

Por otro lado, la ley nacional debería pasar por varias revisiones para incluir plenamente la estrategia del CMCT. Esto resultaría más fácil si Argentina ratifica su firma y participación en él.

Al crear los espacios libres de humo, se estaría protegiendo la vida de los no fumadores y también se realizaría un llamado de atención para los fumadores para que reduzcan su consumo y piensen en dejar de fumar. Es por ello que se recomienda la implementación de leyes provinciales o nacionales de carácter fuerte que no sean permisivas o tengan excepciones.

Conflicto de intereses. Ninguno declarado por los autores.

Declaración. Las opiniones expresadas en este manuscrito son responsabilidad del autor y no reflejan necesariamente los criterios ni la política de la RPSP/ PAJPH y/o de la OPS. 


\section{REFERENCIAS}

1. Frazer K, Callinan JE, McHugh J, van Baarsel S, Clarke A, Doherty K, et al. Legislative smoking bans for reducing harms from secondhand smoke exposure, smoking prevalence and tobacco consumption (Review). Summary of findings for the main comparison. Cochrane Database Syst Rev. 2016;2(CD005992).

2. Barrenechea GG, Cali RS. Mortalidad atribuible al tabaquismo en Tucumán, Argentina 2001-2010. Med. 2016;76(5):287-93.

3. Organización Mundial de la Salud (OMS). Convenio Marco de la OMS para el Control del Tabaco. Rev Esp Salud Publica. 2003;77(4):475-96. Disponible en: http:// www.scielosp.org/scielo.php?script=sci arttext\&pid=S1135-57272003000400005\&lng=es\&nrm =iso\&tlng=es

4. Konfino J, Ferrante D, Mejia R, Coxson P, Moran A, Goldman L, et al. Impact on cardiovascular disease events of the implementation of Argentina's national tobacco control law. Tob Control. 2014;23(2):e6.

5. Lin H, Wang H, Wu W, Lang L, Wang Q, Tian L. The effects of smoke-free legislation on acute myocardial infarction: a systematic review and meta-analysis. Disponible en: https://bmcpublichealth.biomedcentral.com/track/pdf/10.1186/1471-245813-529 Acceso el 9 de junio de 2018.

6. Organización Mundial de la Salud (OMS). Informe OMS sobre la epidemia mundial de tabaquismo. Plan de medidas MPOWER, 2018. Disponible en: http:// apps.who.int/iris/bitstream/handle/10665 / 43897 / $9789243596280 \_s p a$. pdf;jsessionid=DBF96FD1B8540D12881C 3972CD3033CA? sequence $=1$

7. Wang TW, Lemos PR, McNabb S, King BA. Attitudes toward smoke-free public housing among U.S. adults, 2016. Am J Prev Med. 2018;54(1):113-8. Disponible en: http://dx. doi.org/10.1016/j.amepre.2017.08.026

8. Poder Ejecutivo Nacional de la República Argentina. Ley 23344. Disponible en: http:// servicios.infoleg.gob.ar/infolegInternet/ anexos/20000-24999/23223/norma.htm

9. Poder Ejecutivo Nacional de la República Argentina. Ley 26687. Regulación de la publicidad, promoción y consumo de los productos elaborados con tabaco. Disponible en: http://servicios.infoleg.gob.ar/infolegInternet/anexos/180000-184999/183207/ norma.htm

10. Ministerio de Salud de la Nación Argentina. Tercera encuesta nacional de factores de riesgo para enfermedades no transmisibles, 2015. Disponible en: http://www.msal.gob.ar/ images/stories/bes/graficos/0000000544cnt-2015_09_04_encuesta_nacional_factores_ riesgo.pdf Acceso el 9 de junio de 2018.
11. Cummings KM, Hyland A, Borland $\mathrm{R}, \mathrm{Li}$ $\mathrm{Q}$, Yong HH, McNeill A, et al. Individuallevel predictors of cessation behaviours among participants in the International Tobacco Control (ITC) Four Country Survey. Tob Control. 2006;15(S3):83-94.

12. Steele F. Module 5 (concepts): Introduction to multilevel modelling concepts. 2008;5. Dsponible en: http://www.bristol.ac.uk/ media-library/sites/cmm/migrated/documents/5-concepts-sample.pdf

13. Heloma A, Jaakkola MS. Four-year follow-up of smoke exposure, attitudes and smoking behaviour following enactment of Finland's national smoke-free work-place law. Addiction. 2003;98(8):1111-7.

14. Seo DC, Macy JT, Torabi MR, Middlestadt SE. The effect of a smoke-free campus policy on college students' smoking behaviors and attitudes. Prev Med (Baltim). 2011;53(4-5):347-52. Disponible en: http:/ / dx.doi.org/10.1016/j.ypmed.2011.07.015

15. Parks MJ, Kingsbury JH, Boyle RG, Evered S. Household implementation of smoke-free rules in homes and cars: a focus on adolescent smoking behavior and secondhand smoke exposure. Am J Heal Promot. 2018. Disponible en: https://doi. org/10.1177/0890117118776901

16. Kaufman P, Kang J, Kennedy RD, Beck P, Ferrence R. Impact of smoke-free housing policy lease exemptions on compliance, enforcement and smoking behavior: a qualitative study. Prev Med Reports. 2018;10:29-36. Disponible en: https://doi. org/10.1016/j.pmedr.2018.01.011

17. Anyanwu PE, Craig P, Katikireddi SV, Green MJ. Impacts of smoke-free public places legislation on inequalities in youth smoking uptake: study protocol for a secondary analysis of UK survey data. BMJ Open. 2018;8(3):e022490. Disponible en: http://bmjopen.bmj.com/lookup/ doi/10.1136/bmjopen-2018-022490

18. Smith S, Roberts N, Kerr S, Smith S. Behavioral interventions associated with smoking cessation in the treatment of tobacco use. Heal Serv Insights. 2013;79. Disponible en: http://www.la-press.com/ behavioral-interventions-associated-with-smoking-cessation-in-the-trea-article-a3825

19. De la Cruz F. Modelos multinivel. Rev Peru Epidemiol. 2008;12:1-8. Disponible en: http://sisbib.unmsm.edu.pe/bvrevistas / epidemiologia/v12_n3/pdf/a02v12n3.pdf

20. Ministerio de Salud de la Nación Argentina. Encuesta Nacional de Factores de Riesgo 2005. Disponible en: https:// www.indec.gov.ar/ftp/cuadros/menusuperior/enfr/ENFR2005_Baseusuario.rar Acceso el 9 de junio de 2018.
21. Ministerio de Salud de la Nación Argentina. Encuesta Nacional de Factores de Riesgo 2009. Disponible en: http:// www.msal.gob.ar/images/stories/bes/ graficos/0000000553cnt-2014-10_encuesta-nacional-factores-riesgo-2005_informebreve-final.pdf

22. Ministerio de Salud de la Nación Argentina. Encuesta Nacional de Factores de Riesgo 2013. Disponible en: https:// www.indec.gov.ar/ftp/cuadros/menusuperior/enfr/ENFR2013_baseusuario.rar Acceso el 9 de junio de 2018.

23. Bates D, Mächler M, Bolker B, Walker S. Fitting linear mixed-effects models using lme4. Journal of Statistical Software. 2015;67:1. Disponible en: http://arxiv. org/abs/1406.5823

24. Fichtenberg CM. Effect of smoke-free workplaces on smoking behaviour: systematic review. BMJ. 2002;325:188.

25. Mcmillen RC, Winickoff JP, Klein JD. US adult attitudes and practices regarding smoking restrictions and child exposure to environmental tobacco smoke. Pediatrics. 2003;112(1Pt1):e55-60.

26. Gentzke AS, Hyland A, Kiviniemi M, Travers MJ. Attitudes and experiences with secondhand smoke and smoke-free policies among subsidised and market-rate multiunit housing residents living in six diverse communities in the USA. Tob Control. 2018;27(2):194-202.

27. Tworek C, Yamaguchi R, Kloska DD, Emery S, Barker DC, Giovino GA, et al. State-level tobacco control policies and youth smoking cessation measures. Health Policy (New York). 2010;97(2,):136-44.

28. Subramanian V. Patterns and distribution of tobacco consumption in India: cross sectional multilevel evidence from the 1998-9 national family health survey. BMJ. 2004;328(7443):801-6. Disponible en: http://www.bmj.com/cgi/doi/10.1136/ bmj.328.7443.801

29 Peruga A, Hayes LS, Aguilera X, Prasad $\mathrm{V}$, Bettcher DW. Correlates of compliance with national comprehensive smoke-free laws. Tob Control. 2017;tobaccocontrol-2017-053920. doi: 10.1136/ tobaccocontrol-2017-053920.

Manuscrito recibido el 15 de diciembre de 2017. Aceptado para publicación, tras revisión, el 27 de julio de 2018. 
ABSTRACT

Behavioral changes in smokers after implementation of anti-smoking legislation in Argentina

RESUMO

\section{Mudança de comportamento nos fumantes após a implementação da legislação antifumo na Argentina}

Objective. Identify factors related to behavioral changes in smokers over 18 years of age following implementation of provincial policies for $100 \%$ smoke-free spaces in Argentina.

Methods. A cross-sectional analytical study was conducted. The multilevel technique was used with data from different hierarchies. Secondary data sources were used: National Risk Factor Surveys 2005, 2009, and 2013.

Results. The individual analysis of explanatory variables revealed that residents of provinces that do not have smoke-free legislation were $40 \%$ less likely to consider quitting smoking than people who live in provinces with such legislation. In provinces without smoke-free spaces, males over 65 years old are even less likely to think about quitting smoking.

Conclusions. The results suggest that legislation based on Article 8 of the Framework Convention would be effective in promoting tobacco cessation in Argentina.

Keywords Tobacco; behavior; multilevel analysis; health policy; public health; Argentina.

Objetivo. Identificar os fatores associados à mudança de comportamento nos fumantes maiores de 18 anos após a implementação de políticas provinciais de proibição do fumo na Argentina.

Métodos. Foi realizado um estudo analítico de corte transversal. Foi usada uma técnica de modelos multiníveis com dados de diferentes hierarquias. As fontes de dados secundários consultadas foram as iterações de 2005, 2009 e 2013 da Pesquisa Nacional de Fatores de Risco.

Resultados. $\mathrm{Na}$ análise individual das variáveis explicativas, verificou-se que os habitantes das províncias sem legislação antifumo tinham uma probabilidade $40 \%$ maior de não considerar parar de fumar quando comparados aos moradores das províncias com legislação antifumo. Em comparação às províncias com legislação antifumo, nas províncias sem esta legislação, ser do sexo masculino e ter idade acima de 65 anos foram as características associadas a uma maior chance de não considerar parar de fumar.

Conclusões. Os resultados do estudo indicam que uma legislação em conformidade com o Artigo 8 da Convenção-Quadro para o Controle do Tabaco da OMS seria efetiva para incentivar as pessoas a pararem de fumar na Argentina.

Palavras-chave Tabaco; comportamento; análise multinível; política de saúde; saúde pública; Argentina. 